\title{
Differential hippocampal and retrosplenial involvement in egocentric-updating, rotation, and allocentric processing during online spatial encoding: an fMRI study
}

\author{
Alice Gomez ${ }^{1,2,3 *}$, Mélanie Cerles ${ }^{1,2}$, Stéphane Rousset ${ }^{1,2}$, Chantal Rémy ${ }^{4,5}$ and Monica Baciu ${ }^{1,2}$ \\ ${ }^{1}$ LPNC, Université Grenoble Alpes, Grenoble, France \\ ${ }^{2}$ CNRS, LPNC UMR 5105, Grenoble, France \\ ${ }^{3}$ ESPE, Centre de Neurosciences Cognitives, UMR 5229, Université Claude Bernard Lyon 1, Bron, France \\ 4 Joint Service Unit, UMS 3552, 'IRMaGe', CNRS/INSERM, Grenoble Institute of Neuroscience, Joseph-Fourier University, Grenoble, France \\ ${ }^{5}$ Team 5 "Functional Neuroimaging and Brain Perfusion" of Grenoble Institute of Neuroscience, INSERM/CEA, Joseph Fourier University, Grenoble, France
}

\section{Edited by:}

Arne Ekstrom, University of

California, Davis, USA

Reviewed by:

Rebecca Knight, University of Hertfordshire, UK

Hui Zhang, University of Bonn, Germany

\section{${ }^{*}$ Correspondence:}

Alice Gomez, Centre de Neurosciences Cognitives, UMR 5229, 67 Boulevard Pinel, 69675 Bron Cedex, France e-mail: alice.gomez.fr@gmail.com
The way new spatial information is encoded seems to be crucial in disentangling the role of decisive regions within the spatial memory network (i.e., hippocampus, parahippocampal, parietal, retrosplenial,...). Several data sources converge to suggest that the hippocampus is not always involved or indeed necessary for allocentric processing. Hippocampal involvement in spatial coding could reflect the integration of new information generated by "online" self-related changes. In this fMRI study, the participants started by encoding several object locations in a virtual reality environment and then performed a pointing task. Allocentric encoding was maximized by using a survey perspective and an object-to-object pointing task. Two egocentric encoding conditions were used, involving self-related changes processed under a first-person perspective and implicating a self-to-object pointing task. The Egocentric-updating condition involved navigation whereas the Egocentric with rotation only condition involved orientation changes only. Conjunction analysis of spatial encoding conditions revealed a wide activation of the occipito-parieto-frontal network and several medio-temporal structures. Interestingly, only the cuneal areas were significantly more recruited by the allocentric encoding in comparison to other spatial conditions. Moreover, the enhancement of hippocampal activation was found during Egocentric-updating encoding whereas the retrosplenial activation was observed during the Egocentric with rotation only condition. Hence, in some circumstances, hippocampal and retrosplenial structures-known for being involved in allocentric environmental coding-demonstrate preferential involvement in the egocentric coding of space. These results indicate that the raw differentiation between allocentric versus egocentric representation seems to no longer be sufficient in understanding the complexity of the mechanisms involved during spatial encoding.

Keywords: hippocampus, retrosplenial, allocentric, egocentric, navigation, rotation

\section{INTRODUCTION}

In order to interact with space, humans need to integrate spatial information according to new objects encountered and/or new motion information (e.g., object- or self-motion, Wolbers and Hegarty, 2010). Encoding and storing spatial information is supposed to rely on the activity of one of these two types of spatial representation (Burgess, 2006): (a) egocentric, centered on the subject, coding for self-to-object relations; (b) allocentric, centered on the environment, coding for object-to-object relations. Various evidence sources, ranging from single neuron recordings in animals (O'Keefe and Dostrovsky, 1971; Andersen et al., 1985) to fMRI and neuropsychological observations (Bisiach and Luzzatti, 1978; Aguirre and D’Esposito, 1999; Holdstock et al., 2000; Mellet et al., 2000; Spiers et al., 2001; Chokron, 2003; Burgess et al., 2006; Fields and Shelton, 2006; Maguire et al., 2006; Shrager et al., 2008), suggest that the two types of spatial representations rely on the occipito-temporo-parietal cortices. Among these regions, the posterior parietal cortex sustains egocentric processing (Chokron, 2003) while the medio-temporal regions are required for the allocentric processing (O'Keefe and Nadel, 1978; Holdstock et al., 2000; Milner and Goodale, 2008; Finke et al., 2011).

The connection proposed between the allocentric processing and the hippocampus is partly due to the strong relationship between the "Place cells" firing and landmark sensory aspects observed in animal studies (e.g., O'Keefe and Dostrovsky, 1971; O'Keefe and Nadel, 1978; O'Keefe and Burgess, 1996). fMRI studies in humans during route navigation congruently revealed that the activation of hippocampal and parahippocampal regions correlated with relevant landmark information (Janzen and Van Turennout, 2004; Janzen and Weststeijn, 2007; Janzen et al., 2007). However, several data sources suggest that the hippocampus is not always involved or necessary for allocentric processing (Aguirre et al., 1996; Bohbot et al., 1998; Galati et al., 2000; 
Committeri et al., 2004; Bastin et al., 2013). For instance, Bohbot et al. (1998) reported, that the performance of patients suffering from unilateral hippocampal lesions in an adapted version of the Morris water maze task (assessing landmark-based allocentric representation) was similar to that of control subjects. Moreover, as revealed by $\mathrm{fMRI}$, some variants of allocentric processing seem to be supported by a large network of regions including parietal, retrosplenial, and parahippocampal cortices (e.g., Aguirre et al., 1996; Vallar et al., 1999; Galati et al., 2000; Committeri et al., 2004; Zhang and Ekstrom, 2013; for review Galati et al., 2010).

The observable discrepancy between these results and the exclusive allocentric role proposed for the medio-temporal lobe (O'Keefe and Dostrovsky, 1971; O'Keefe and Nadel, 1978) could be due to the way spatial information is encoded. Specifically, beyond the allocentric vs. egocentric distinction relative to the type of spatial relation involved (object-to-object or self-toobject, respectively), the presence or the absence of self-related changes could be a decisive factor in the hippocampal involvement. Using a short-term spatial task, Gomez et al. (2014, 2012) disentangled different sources of information required during encoding. The authors showed that patients suffering from bilateral hippocampal atrophy were impaired for reproducing a trajectory learned on the basis of self-motion information. However, they performed similarly to controls when the trajectory was learned without any self-related changes (i.e., observing the experimenter producing the trajectory). This result is in-line with the hypothesis that hippocampal activity may be observed when self-related changes are required to build spatial representations.

Functional MRI evidence provides indirect support to this last hypothesis. Studies manipulating the type of visual perspective during spatial encoding itself showed that hippocampal and parahippocampal structures were more activated during route navigation than during survey navigation (Shelton and Gabrieli, 2002; Shelton and Pippitt, 2007). Hence, despite previous behavioral reports of a strong connection between survey perspective and allocentric representation formation (Thorndyke and HayesRoth, 1982; Allen, 1999), such processing seems less tightly related to hippocampal activity than during route navigation. The enhanced activation of hippocampal and parahippocampal structures for route navigation may reflect the integration of new information generated by self-related changes during the egocentric spatial processing (Shelton and Pippitt, 2007).

Beyond the type of visual perspective during spatial encoding, the participants' spatial processing during each encoding condition should be further controlled to insure that the differential hippocampal activity is related to new self-related changes. Actually, the foreseen retrievals tasks can modulate the strategies (allocentric vs. egocentric) used by participants during encoding itself (Shelton and Gabrieli, 2004). From a methodological point of view, this control can be achieved by warning participants that they will have to perform an object-to-object relation retrieval of the spatial information before they enter the survey perspective encoding. On the contrary, participants can be instructed to encode spatial information to subsequently perform a selfto-object relation retrieval task which will insure that the two tasks will in fact differ on the integration (or not) of self-related changes.
If the presence/absence of self-related change is indeed crucial, the information about how the self-related changes are generated could be relevant in providing a thorough understanding of the mechanisms responsible for the different involvements in the spatial memory network. Specifically, given areas could be involved when a change in head direction modifies orientation cues without updating the observer's location. On the reverse, different ones could be involved when both a change in orientation cues and an update in the observer's position occur.

Regarding the head orientation, studies on single cell recordings in animals revealed that several cells fired selectively when the animal oriented its head in a given direction. Cells' localization was first described in post-subiculum (Ranck, 1984), limbic system (Taube, 1998; Robertson et al., 1999) including the retrosplenial cortex (Cho and Sharp, 2001), and hippocampus (Leutgeb et al., 2000) of animals. In humans, heading disorientation was observed after lesion of the retrosplenial cortex (Aguirre and D’Esposito, 1999). More recently, using single-neuron recordings, Jacobs et al. (2010) showed that human entorhinal cortex could contain path cells encoding in a clockwise or counterclockwise route direction during navigation in circular environments. Functional MRI studies have revealed that retrosplenial activity was modulated by previously learnt variations of the head direction (Baumann and Mattingley, 2010).

Farrell and Robertson (1998) defined as the egocentricupdating processing, changes related to both head orientation (i.e., head direction changes) and observer's spatial position. Several animal studies and models of spatial neuronal networks have involved hippocampal "Place cells" during such spatial changes. In fact, based on neuronal recordings in animals, these models indicate that place cell firing is also related to path integration (Mittelstaedt and Mittelstaedt, 1982), which refers to the ability to return directly to a starting point from any location relying mainly on idiothetic cues (i.e., self-motion cues). Path integration can be considered as a subtype of the general process of egocentric-updating where objects are updated relative to the individual (Burgess, 2008). These models emphasized the need to combine landmark sensory aspects and idiothetic cues to build a place code (e.g., Redish and Touretzky, 1997; McNaughton et al., 2006).

These various approaches suggest that the retrosplenial cortex may be crucial in processing heading changes within egocentric referencing, while the hippocampus may be related to egocentricupdating. No fMRI evidence in humans has clearly disentangled the underlying network subtending these two types of processing.

In the present fMRI study, we aim to characterize the neural substrate of spatial memory encoding. Beyond an allocentric (object-to-object) vs. egocentric (self-to-object) distinction, we further disentangled the egocentric information by varying the types of self-related changes during encoding (egocentricupdating vs. rotation only). As such, we defined three spatial encoding conditions: Allocentric, Egocentric-updating, and Egocentric with rotation only.

One hypothesis is that the cerebral organization evolution of cognitive functions from animals to humans could lead to a different neural specialization of spatial memory encoding in specific areas such as the hippocampal and retrosplenial areas. 
Alternatively, given the neuropsychological evidence, we expect that spatial cognitive functions share similar structures in animals and humans. Therefore, we can expect that the egocentricupdating condition reveals additional activity in the hippocampal structure compared to the allocentric condition. This supports the idea that the human hippocampal area could also code for self-related changes. Additionally, compared to egocentricupdating, the egocentric with rotation only condition should involve retrosplenial activation, supporting the idea that the human retrosplenial cortex codes specifically for orientation changes.

\section{MATERIALS AND METHODS}

The experiment consisted of two phases: a training phase performed outside the MR magnet and a testing phase performed during the MR examination. The training phase had three objectives: (1) familiarization with the virtual presentation via free navigation in the environment, (2) instructions on how to perform each task, (3) task execution with performance feedback. During the testing phase, three spatial encoding conditions and a control condition were performed during a block-design paradigm.

\section{PARTICIPANTS}

Eighteen adults (age range 17-30, average age 23.5 SD 2.5, 13 males) took part in the experiment. All participants were right-handed according to the Edinburgh Handedness Inventory (Oldfield, 1971). They had normal or corrected-to-normal vision and no history of neurological or psychiatric disorders. They gave their informed written consent for the experiment and the study was approved by the local ethic committee (CPP $\mathrm{n}^{\circ} 08-\mathrm{CHUG}-10$, 20/05/2008).

\section{SPATIAL ENVIRONMENT, SPATIAL LAYOUTS, AND ENCODING FILMS}

A Virtual Reality Markup Language (VRML) was used to create the spatial environment. This virtual environment was a $9 \times 9 \times$ $3 \mathrm{~m}$ room with stone walls. Tile flooring for the southern half and wood flooring for the northern half provided easy orientation in the environment. Each environment contained 6 objects. Twenty different spatial layouts were created by randomly changing the 6 objects' positions. Spatial layouts presenting familiar configurations such as lined-up objects were removed. An in-house VRML-Prime software was created (http://webu2.upmf-grenoble.fr/LPNC/membre_eric_guinet) with the following characteristics: (1) joystick-navigation in the environment, (2) online joystick data recording, and (3) joystick-data-based feedback. To control what the participants see, the VRML-Prime was used to create films of the pre-determined layouts. VRML-Prime made it possible to switch independently between: (1) the visual perspective of the environment (aerial or ground-level) and (2) the type of camera movement in the environment (i.e., rotation only, route navigation, or sequential map presentation).

Three visual spatial encoding conditions were created (See Figure 1): Allocentric (A), Egocentric-updating (EU), and Egocentric with Rotation Only (ERO). For the A films (See Video 1, Gomez, 2014a, http://figshare.com/articles/Allocentric_ video_example/902846), a survey perspective (i.e., a bird's eye perspective, looking straight down, with $15 \%$ of the environment visible at any moment) was adopted; the camera scanned the map of the environment with an unchanging orientation. This viewpoint was selected to allow the average amount of environment visible at any given point to be equivalent to the ground-level condition. In addition, pilot studies have indicated that this viewpoint induced participants to spontaneously perceive this as "the map" condition. For the EU films (See Video 2, Gomez, 2014b, http://figshare.com/articles/Egocentric_updating_video_example/ 902847), a ground-level 1st person perspective was adopted; the camera movement was used to simulate the view of an observer walking through the environment. For ERO films (See Video 3, Gomez, 2014c, http://figshare.com/articles/Egocentric_ with_Head_rotation_video_example/902848), a ground-level 1st person-perspective (i.e., looking straight from the perspective of a $1 \mathrm{~m} 80$ tall observer) was adopted; the camera movement offered a rotation of $180^{\circ}$, from a fixed location (i.e., one side of the room). Each one of the 20 spatial layouts, resulted in three encoding films that lasted $17700 \mathrm{~ms}$. A fourth control category of films was created using a mix of the ERO, EU, and A films: about $6 \mathrm{~s}$ of each of the three films were selected and pooled together in a random order, thus resulting in a $17700 \mathrm{~ms}$ control film. The camera movement simulated a path of about $20 \mathrm{~m}$ with one or two direction changes. Given that self-motion perception in virtual environments is most accurate when displacement velocity resembles natural locomotion, we adopted a speed of a moderately paced walk (approximately $1.5 \mathrm{~m} / \mathrm{s}$ ). The path and speed for the aerial and ground films were the same. The layout configuration presented in each movie was always different and contained an average of 5 objects (range 4-6, as all 6 objects from an environment were not visible in each movie). The encoding conditions for a given spatial layout were randomly assigned for each participant.

\section{EXPERIMENTAL CONDITIONS: THE SPATIAL TASKS}

Each spatial task trial was composed of an encoding and an immediate test phase (See Figure 1).

In the encoding phase, a film (one of the four types A, EU, ERO, and control, C) was presented to the participants and they were asked to encode and update with the sequential presentation the objects' positions of the objects in the environment. The A film presented a survey perspective of the environment, the ERO movie presented a 1st person rotation from an unchanged location, the EU movie presented a 1st person perspective navigation in the environment and the $\mathrm{C}$ movie was a mixture of the three spatial movies which made the control for purely visual stimulations possible. The film presentation was preceded by a $300 \mathrm{~ms}$ fixation point included in the "encoding phase." Participants' spatial knowledge was then tested to control for attention and spatial processing during the encoding phase.

In the test phase, participants were instructed to locate the direction of an object, and to point in its direction with the joystick when prompted. To enhance each type of spatial processing during the encoding phase, participants were told beforehand which test will follow. In the A condition, the A referencing was maximized by asking participants to point in the direction of an object relative to another object in the fixed referencing of the 


\section{Survey perspective (Map-like) \\ Allocentric

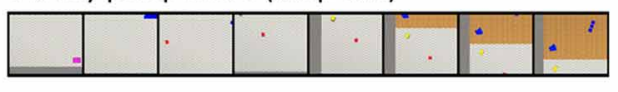

1-st person ground level perspective

\section{Egocentric \\ updating}

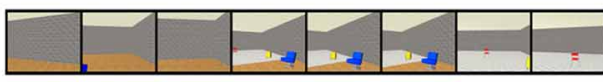

Egocentric rotation only

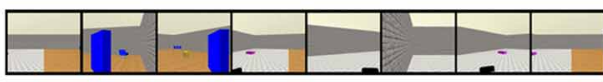

Mix of perspectives

Control

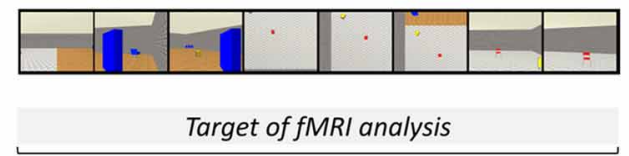

1. Spatial encoding phase

(18s.)

\section{Object-to-object pointing

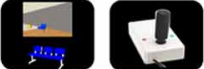

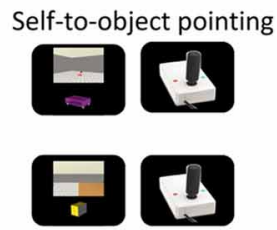

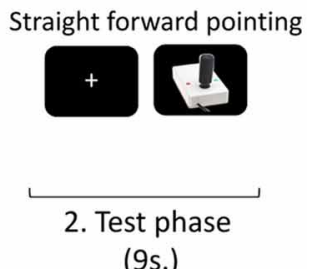

(9s.)
FIGURE 1 | A trial of each experimental condition performed during the experimental phase inside the MR imager is presented. Each of the four conditions, A, Allocentric; EU, Egocentric-updating; ERO, Egocentric with Rotation Only; and C, Control; was divided into an encoding phase and a test phase. In the encoding phase, participants saw a presentation of the environment's layout: (A) from a survey perspective, (EU) from a 1-st person ground level perspective with navigation, (ERO) from a 1-st person ground level perspective with a rotation only (C) with a mix of perspectives. In the test phase, participants had to point in the direction of an object using a joystick: (A) from the location of another object, ( $E U$ and ERO) from their body position; in condition C, they pointed straightforward. environment (i.e., object-to-object pointing relative to the fixed orientation of the map). Participants were instructed to imagine that they were sketching the direction on the map of the environment. The egocentric referencing was maximized by asking participants to point in the direction of an object relative to their position (i.e., self-to-object pointing), in the EU and ERO conditions.

Each test phase of a trial lasted $9 \mathrm{~s}$ : first a question screen was presented during $6 \mathrm{~s}$, and then a joystick picture was presented during $3 \mathrm{~s}$. The joystick picture $(320 \times 256$ pixels $)$ presented on a black screen, served to prompt the participants' response and to collect behavioral performance. The question screen presented (1) the origin of the spatial referencing and (2) the object-tobe-pointed-to on a black background. The origin of the spatial referencing was presented by a 1st person point of view of the environment in the egocentric referencing. The participant could thus simulate its position in the environment from the perspective seen (the point of view was extracted from the previous film). In the A referencing, the point of view was replaced by the presentation of a second object with contextual information from the background. Both the origin and the object-to-be pointed-to were selected from the first and second half of the film respectively, and separated by a minimum of $9 \mathrm{~s}$ in the film presentation. They were presented centered on a black background, with the origin at the top $(896 \times 670$ pixels picture $)$, and the objectto-be-pointed-to at the bottom $(512 \times 410$ pixels picture). For condition $\mathrm{C}$, the test was aimed to control for motor preparation, and participants were asked to point straight in front of them (i.e., $0^{\circ}$ angle).

\section{EXPERIMENTAL PROCEDURE}

As mentioned previously, the experimental procedure was composed of two phases: a training phase outside the magnet and an experimental phase during fMRI.

\section{Training phase outside the MR imager}

Participants were first asked to navigate freely within an environment designed for this phase using the VRML-prime software. The purpose of this step of the training procedure was to familiarize participants with the use of the joystick, the virtual desktop presentation and the 6 objects.

Then, participants were trained in the experimental conditions previously described. An example of each spatial task was presented (A, EU, ERO, and C), and participants were instructed how to perform each of the four tasks. During the training phase, the test phase of a trial was completed by a visual feedback on the pointing response (from online data recording). In the feedback of the A training condition, the feedback screen displayed the entire map of the environment, with (1) a green arrow (i.e., correct answer) pointing from the origin object toward the expected target object and (2) a blue arrow (i.e., given answer) pointing from the origin object toward the given direction. The error-angle was thus shown as the angle between each of these arrows. The smaller the angle, the more precise the response. This feedback procedure proved successful since all participants were subsequently able to perform the tasks with relatively low pointing errors. In the feedback of the EU and ERO training conditions, a 1st-person perspective video was presented, showing a rotation from a fixed location. The camera was placed on the original 
location facing the given answer direction. It then rotated until it was in line with the direction of the expected answer. It thus provided an angular distance between the correct and the given answer. Thus, the bigger the error-angle was, the greater the video rotation. We performed training to eliminate learning and habituation effects. Participants were trained using 10 trials of each spatial task.

\section{Experimental phase inside the MR imager}

Procedure. The participants were told that they had to perform the same tasks as before but in the scanner. The trials were displayed using E-prime software (E-prime Psychology Software Tools Inc., Pittsburgh, PA, USA) synchronized via the signals from the scanner. A back-projection screen, received the images, and a mirror, attached to the head coil, allowed the participant to view the screen. After being positioned within the magnet, participants were shown an example of each trial performed during the training phase without concurrent fMRI recording to familiarize them with performing the task while in a horizontal position. Participants indicated their response on an MR-compatible joystick that was positioned on the upper part of the right leg. Participants moved the joystick with the right hand and pushed a button with the left hand when the joystick was oriented in the desired direction. Angle errors were recorded during the test phase using VRML-prime. Participants were not given any feedback about their performance. All participants were presented with the spatial tasks in the same block order as in the training phase (e.g., A, ERO, EU, and C, counterbalanced across participants). When grouping the encoding and test phase, each trial lasted $27 \mathrm{~s}$ with an inter-trial interval of $3 \mathrm{~s}$. Participants underwent two functional scans (of four blocks each) with five trials of each conditions. The duration of the functional scan was thus $9 \mathrm{~min}$, following a brief period during which the scanner reached equilibrium. In this phase, five trials of each condition were consecutively presented in a random order and formed a block. The order of the blocks was repeated twice for each participant. The block order was counterbalanced across participants. For each functional scan, 200 functional volumes were acquired.

MR acquisition. The experiment was performed in a whole-body 3T MR scanner (Bruker MedSpec S300) with $40 \mathrm{mT} / \mathrm{m}$ gradient strength. For functional scans, the manufacturer-provided gradient-echo/T2* weighted EPI method was used. Thirty-nine adjacent axial slices parallel to the bi-commissural plane were acquired in the interleaved mode. Slice thickness was $3.5 \mathrm{~mm}$. The in-plane voxel size was $3 \times 3 \mathrm{~mm}(216 \times 216 \mathrm{~mm}$ field of view acquired with a $72 \times 72$ pixels data matrix; reconstructed with zero filling to $128 \times 128$ pixels). The main sequence parameters were: $T R=3 \mathrm{~s}, T E=30 \mathrm{~ms}$, flip angle $=77^{\circ}$. To correct images for geometric distortions induced by local B0-inhomogeneity, a B0 fieldmap was obtained from two gradient echo data sets acquired with a standard $3 \mathrm{D}$ FLASH sequence $(\triangle T E=$ $9.1 \mathrm{~ms}$ ). The fieldmap was subsequently used during data processing. Finally, a T1-weighted high-resolution three-dimensional anatomical volume was acquired, by using a 3D Modified Driven Equilibrium Fourier Transform (MDEFT) sequence (field of view $=256 \times 224 \times 176 \mathrm{~mm}$; resolution: $1.333 \times 1.750 \times$
$1.375 \mathrm{~mm}$; acquisition matrix: $192 \times 128 \times 128$ pixels; reconstruction matrix: $256 \times 128 \times 128$ pixels).

Data processing. Data analysis was performed by using the general linear model as implemented in SPM5 (Welcome Department of Imaging Neuroscience, London, UK, www.fil.ion. ucl.ac.uk/spm) where each event was modeled using a hemodynamic function model. Data analysis started with the spatial pre-processing steps. First, the functional volumes were timecorrected (slice timing) with the 19th slice as the reference, in order to correct for effects induced by the different acquisition time of each slice within the functional volume. Subsequently, all volumes were realigned to correct for head motion using rigid body transformations. After discarding the first four slices, while the scanner reached equilibrium, the first volume of the first ERfMRI session was taken as the reference volume (i.e., this volume was originally the fifth volume). Unwarping was performed by using the individually acquired fieldmaps, to correct for interaction between head movements and EPI distortions. T1-weighted anatomical volume was co-registered to mean images created by the realignment procedure and was normalized to the MNI space using a trilinear interpolation. The anatomical normalization parameters were subsequently used for the normalization of functional volumes. Finally, each functional volume was smoothed by an 8-mm FWHM (Full Width at Half Maximum) Gaussian kernel to improve differences in intersubject localization. Time series for each voxel were high-pass filtered $(1 / 128 \mathrm{~Hz}$ cutoff) to remove low frequency noise and signal drift.

Statistical analysis of neuroimaging data. After spatial preprocessing steps, the statistical analysis was first performed separately on the functional images acquired for each task during the first $18 \mathrm{~s}$ of each trial (reflecting the encoding phase of a trial). The cerebral activity during the test phase of each trial was not included in the present study, thus, the functional images from the test phase were discarded from the analysis. The conditions of interest ( $\mathrm{A}, \mathrm{EU}, \mathrm{ERO}$, and $\mathrm{C}$ ) were modeled as 4 regressors convolved with a canonical hemodynamic response function (HRF). The movement parameters derived from the realignment corrections ( 3 translations and 3 rotations) were also entered in the design matrix as additional factors. The trial performance (i.e., error angle) was entered as a parametric modulator of each trial. The general linear model was then used to generate the parameter estimates of the activity for each voxel, each condition and each participant. Statistical parametric maps were generated from linear contrasts between the HRF parameter estimates for the different experimental conditions. The spatial resolution of the statistical parametric maps was the same as the spatial resolution of the functional MR acquisition $(3 \times 3 \times 3.5 \mathrm{~mm})$. Several statistical analysis have been performed as follows: Approximate AR(1) autocorrelation model estimated at omnibus $F$-significant voxels $(p<0.001)$, was used over the whole brain. Specific effects were tested with the appropriate linear contrasts of the parameter estimations, and the corresponding contrast images were subsequently entered into a random effects analysis.

Group analysis was performed with the contrast images provided by the individual analyses (Friston et al., 1998). The random 
effects analysis at the group level can be divided as follows depending on the theoretical question underlying the analysis:

- Global spatial encoding network

First, the main contrasts assessed the correlates of spatial encoding for each spatial condition $\mathrm{A}, \mathrm{EU}, \mathrm{ERO}$ vs. the $\mathrm{C}$ condition. Then, the conjunction analysis assessed the neural correlates common to all spatial encoding tasks. For this purpose, we applied an inclusive masking for all three main contrasts (Friston et al., 2005).

- Differenciating neural correlates of spatial encoding conditions First, the main contrasts assessed the questions of interest in this study. EU vs. A provides cerebral substrate engaged by egocentric referencing and due to self-motion information (i.e., idiothetic). A vs. EU provides information about landmark-based information (i.e., allothetic). ERO vs. EU provides information related to the cerebral substrate of rotation processing while EU vs. ERO reveals cerebral network of the egocentric-updating due to self-motion information. We also calculated the contrasts: ERO vs. A and A vs. ERO.

Subsequently, a multiple regression analysis was performed. Specifically, we included individual contrast images (one image per participant) reflecting the mean activation for each previously mentioned contrasts.

Moreover, we also performed a correlation analysis to assess the task-specific regions modulated by the performance. For this purpose, we calculated correlations between the BOLD signal in task-specific regions and the performance of task-execution. This analysis is supposed to reflect the regions which were closely related to spatial processing (Maguire et al., 1998; Wolbers and Buchel, 2005; Wolbers et al., 2007, 2008; Baumann et al., 2010). The evaluated performance was the average angle error size (i.e., difference between the expected pointing direction and the observed pointing direction). The average error size for each participant in each condition was the variable included in a multiple regression analysis. As the correct performance is reflected by the small size of the angle error, the regions showing anti-correlation between BOLD and performance, were considered specifically related to the achievement of spatial processing.

For all our analyses, we used an extent threshold of 10 contiguous voxels, and a voxel-level height threshold of $p<0.001$, uncorrected for contrasts (height threshold: $T=3.55$ ). The extended threshold of 10 voxels was determined empirically and then used for all contrasts. However, as advised by Bennett et al. (2009) FDR threshold are provided in the Tables to provide corrected values detailing the prevalence of false positives. An in-house modification of the "spm_list.m" file, including a nonlinear transform of MNI to Talairach (http://imaging.mrc-cbu. cam.ac.uk/imaging/MniTalairach), allowed us to visualize both MNI and Talairach coordinate in the SPM result. From the displayed Talairach coordinate, anatomical locations were determined using Talairach Daemon software (http://www.talairach. org/, see also Lancaster et al., 2000), and checked with the Talairach and Tournoux paper atlas (Talairach and Tournoux, 1988).
Analyses were also performed with small volume correction (SVC) for multiple comparisons $(P<0.05$, corrected) in ROIs. In this study, we were particularly interested in the role of the hippocampus and retrosplenial areas. Therefore, predefined anatomic labels (WFU PickAtlas Tool, http://www.fmri.wfubmc. edu/download.htm) were applied to identify each of the following ROI: hippocampus (left and right) and Brodmann area 30, 31 (left and right).

\section{RESULTS \\ BEHAVIORAL RESULTS}

Table 1 summarizes the performance values obtained for each condition. An ANOVA conducted on the angular error with conditions as a within-subject variable (A, EU, ERO, C) showed a main effect for experimental conditions $\left[F_{(3,51)}=35.2, M S E=\right.$ 121.64, partial $\left.\eta^{2}=0.67, p<0.001\right]$. Specific contrast of interest A vs. EU, EU vs. ERO, and ERO vs. A were not statistically significant $\left[F_{(1,17)}=1.76, M S E=98.63 . p=0.20\right.$, and $F_{(1,17)}=$ 3.60, $M S E=186.9 . \quad p=0.07$, and $F_{(1,17)}=1.15, \quad M S E=$ 143.06. $p=0.30$, respectively]. Post-hoc Bonferroni comparisons only showed that smaller pointing errors were made in the $\mathrm{C}$ condition (mean $=3.4^{\circ}, S D=3.5^{\circ}$ ) compared to all other spatial conditions $\left(\right.$ mean $=33.4^{\circ}, S D=13.5^{\circ}, p s<0.001$, all other $p$ s are non-significant).

A correlation matrix of spatial performance for each spatial condition revealed a significant correlation between performance values in A and EU conditions $(r=0.5, p<0.05)$. Participants who performed well in the A condition were also good performers in the EU condition. However, the ERO performance was not significantly correlated with A or with EU performance which suggests that those who performed well in the A or EU condition did not necessarily perform well in the ERO condition.

\section{fMRI RESULTS}

\section{Global spatial encoding network}

Allocentric (A) encoding. As illustrated in the Supplementary Material file-Table S1, the contrast A vs. C revealed a large cluster of 4128 activated voxels with an activation peak located within the left cuneus (BA 17). The activation included the bilateral temporal lobe, the bilateral hippocampus, the bilateral parietal lobe (superior parietal lobule, BA 7 and inferior parietal lobule, BA 40), frontal areas (BA 4), and the cerebellum.

Table 1 | Average angle size error (with standard deviations in parentheses) in the object pointing task for each condition.

\begin{tabular}{lc}
\hline $\begin{array}{l}\text { BEHAVIORAL PERFORMANCE } \\
\text { Condition }\end{array}$ & Average error angle size (standard deviation) \\
\hline Allocentric & $33.34(12.01)$ \\
Egocentric & \\
Updating & $37.73(15.48)$ \\
Rotation only & $29.08(11.95)$ \\
Control & $3.36(3.37)$
\end{tabular}

The A condition (with an object-to-object task), the EU and the ERO conditions (with a self-to-object task), and the $C$ condition (with a straightforward pointing task). 
Egocentric-updating (EU) encoding. As illustrated in the Supplementary Material file-Table S2, the contrast EU vs. C revealed a large cluster of 4086 activated voxels with an activation peak within the left hippocampus. Other activations were observed in the bilateral parietal lobe (superior parietal lobule, BA 7 and inferior parietal lobule, BA 40), cingulate gyrus (BA 31), the right frontal area (BA 4), and the cerebellum.

Egocentric with Rotation Only (ERO) encoding. As illustrated in the Supplementary Material file-Table S3, the contrast ERO vs. C revealed a large cluster of 5381 activated voxels with an activation peak located within the left cuneus (BA 17), extending within the bilateral temporal lobe. Other activations were observed in the bilateral parietal lobe (precentral gyrus, BA 4; gyrus-post-central, BA 2, 3), the frontal areas (BA 4,6), and the cerebellum.

Conjunction analysis. The conjunction analysis identified the common activation (Table 2) of spatial encoding. They were located in the right cuneus, BA 18, right hippocampus and left superior parietal lobule, BA 7.

\section{Differenciating neural correlates of spatial encoding conditions}

The following contrasts revealed activation for the spatial condition, without considering the retrieval performance of subjects.

Egocentric-updating compared to Allocentric condition (Table 3, Figure 2). EU vs. A revealed activation in bilateral frontal regions (such as the superior and middle frontal gyri, BA 6, 8, 9, 32, 46), left thalamus, and bilateral cerebellum. More importantly, we observed that the right hippocampus was specifically activated for EU vs. A.

$A$ vs. $E U$ revealed an activation of the bilateral cuneus (BA 18, 19).

We then tested our a-priori hypotheses about a differential hippocampal activation pattern for both conditions (FDR small-volume corrected, $p<0.05$ ) and observed that the right hippocampus was activated in the EU condition (Table 6).

Table 2 | Activated regions for spatial encoding commonly activated for A, EU, ERO, vs. C (Conjunction analysis, statistical threshold: uncorrected $p<0.001$, cluster extent: $k \geq 10$ voxels).

\begin{tabular}{|c|c|c|c|c|c|c|c|c|}
\hline Cerebral activated regions & Side & BA & $\boldsymbol{k}$ & Talair & oordi & $y, z)$ & $T$-value & FDR corrected threshold \\
\hline \multicolumn{9}{|l|}{ OCCIPITAL CORTEX } \\
\hline Cuneus & $\mathrm{R}$ & BA 18 & 3062 & 18 & -96 & 11 & 6.31 & 0.000 \\
\hline Hippocampus & $\mathrm{R}$ & - & 125 & 18 & -32 & 5 & 5.86 & 0.001 \\
\hline \multicolumn{9}{|l|}{ PARIETAL CORTEX } \\
\hline Superior parietal lobule & L & BA 7 & 108 & -27 & -55 & 58 & 4.56 & 0.001 \\
\hline
\end{tabular}

The Talairach coordinates $(x, y, z)$ are indicated for each voxel. The side, Right $(R)$ and Left (L), gyri and Brodmann areas (BA) are mentioned. Abbreviation: A,

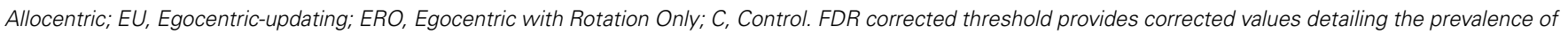
false positives in the analysis.

Table 3 | Activated regions due to self-related changes in location and orientation during spatial encoding, provided by the contrast: (1) EU vs. A for the upper part of the table; (2) A vs. EU for the lower part of the table (random-effect analysis, uncorrected $p<0.001, k \geq 10$ voxels).

\begin{tabular}{|c|c|c|c|c|c|c|c|c|c|}
\hline Contrasts & Cerebral regions & Side & BA & $\boldsymbol{k}$ & Talair & h coor & $(x, y, z)$ & $T$-value & FDR corrected threshold \\
\hline \multirow{7}{*}[EU>A]{} & Hippocampus & $\mathrm{R}$ & - & 11 & 33 & -23 & -8 & 7.08 & 0.137 \\
\hline & \multicolumn{9}{|l|}{ Frontal cortex } \\
\hline & Superior frontal gyrus & $L$ & BA 6 & 11 & -24 & -11 & 68 & 5.85 & 0.137 \\
\hline & Middle frontal gyrus & $\mathrm{R}$ & BA 6 & 14 & 50 & 2 & 42 & 4.82 & 0.134 \\
\hline & Uvula & $\mathrm{R}$ & - & 13 & 33 & -62 & -23 & 6.67 & 0.134 \\
\hline & Declive & $L$ & - & 25 & -12 & -77 & -29 & 5.46 & 0.058 \\
\hline & Thalamus & $\mathrm{L}$ & - & 21 & -3 & -3 & 3 & 6.42 & 0.058 \\
\hline \multirow[t]{2}{*}[A>EU]{} & \multicolumn{9}{|l|}{ Occipital cortex } \\
\hline & Cuneus & $L, R$ & BA 19 & 105 & 0 & -89 & 33 & 4.63 & 0.000 \\
\hline
\end{tabular}

The right hippocampal activation in the EU condition is highlighted. The Talairach coordinates $(x, y, z)$ are indicated for each voxel. The side, Right (R) and Left (L), gyri and Brodmann areas (BA) are mentioned. Regions reported in both contrasts are highlighted. FDR corrected threshold provides corrected values detailing the prevalence of false positives in the analysis. 


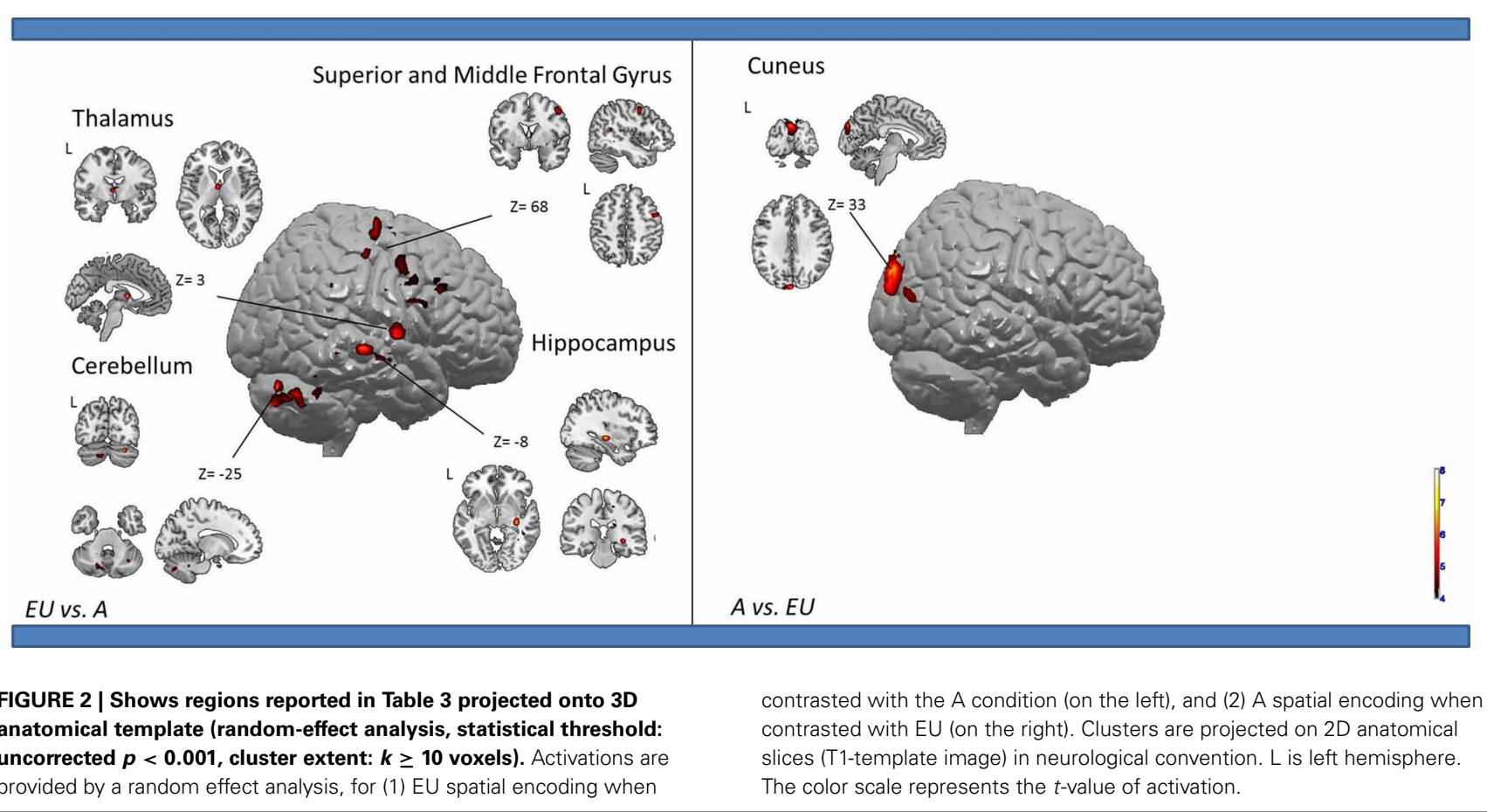

Egocentric with Rotation only compared to Egocentric-updating condition (Table 4, Figure 3). ERO vs. EU activated the right retrosplenial cortex (BA 30), superior parietal lobule (BA 7). Supplementary activity was observed in the bilateral middle and medial frontal gyrus (BA6, 32), right thalamus, and bilateral cerebellum.

$E U v s . E R O$ revealed an activation of the right hippocampus (8 voxels), left superior parietal lobule (BA 5), and right middle and superior temporal gyrus (BA 21,22). Supplementary activity was observed in the bilateral precentral gyrus (BA 4) and cerebellum.

We then tested our a-priori hypotheses about a differential retrosplenial and hippocampal activation pattern for both conditions (FDR small-volume corrected, $p<0.05$ ). We observed that the right Brodmann area 30 was activated in the ERO condition while the right hippocampus was activated in the EU condition (Table 6).

Allocentric compared to Egocentric with Rotation only condition (Table 5, Figure 4). A vs. ERO also revealed activation of the bilateral cuneus (BA 18, 19). Supplementary activations were observed in the left superior temporal gyrus (BA 34, 38), right superior (BA 5) and inferior (BA 40) parietal lobule, bilateral frontal (pre-central gyrus, BA 4), and right cerebellum.

$E R O$ vs. A revealed specific activation in the right retrosplenial cortex (BA 30) extended to the bilateral posterior cingulate (BA 31) and left inferior parietal lobule (BA 40). Supplementary activities were observed in the bilateral frontal regions (superior and middle frontal gyri, BA 6, 8, 9, 32, 46), left thalamus, and bilateral cerebellum.

We then tested our a-priori hypotheses about a differential retrosplenial activation pattern for both conditions (FDR small-volume corrected, $p<0.05)$. We observed that the right
Brodmann area 30 and Brodmann area 31 were activated in the ERO condition (Table 6).

Correlation analysis. Among the A-specific regions, no regions were anticorrelated with the pointing errors. Among the EUspecific regions, only the junction between the right hippocampus and caudate nucleus (peak coordinates: $21,-40,8, T=5.47$, see Figure S1 from Supplementary Material) was significantly anticorrelated with the pointing errors $(r=-0.87)$. A smallvolume correction analysis (FWE corrected, $p<0.05$ ) on the right hippocampus confirmed that the right hippocampus was significantly correlated (peak coordinates: 16, $-33,11$ ). Finally, among the ERO-specific regions, no regions were anticorrelated with the pointing errors.

\section{DISCUSSION}

Our results confirmed the activation of a large occipito-parietotemporal network common for spatial encoding conditions (e.g., Ghaem et al., 1997). Beyond the classical allocentric-egocentric distinction, the type of referencing induced by the visual perspective during encoding and the task-demand, allowed the identification of essential regions within the spatial memory network. The major result was the specific involvement of two areas during the self-related conditions, which were the retrosplenial cortex for orientation changes, and the right hippocampus for self-location changes.

The first crucial result of the study reveals an enhanced retrosplenial activity when contrasting ERO to EU. Encoding new spatial information during the ERO condition only relied on rotation changes, while, during the EU condition, the weight of rotation information during the integration of new spatial information was weakened by the concomitant change of location. 
Table 4 | Activated regions for differential effects within two types of Egocentric spatial encoding, rotation changes vs. orientation and location changes.

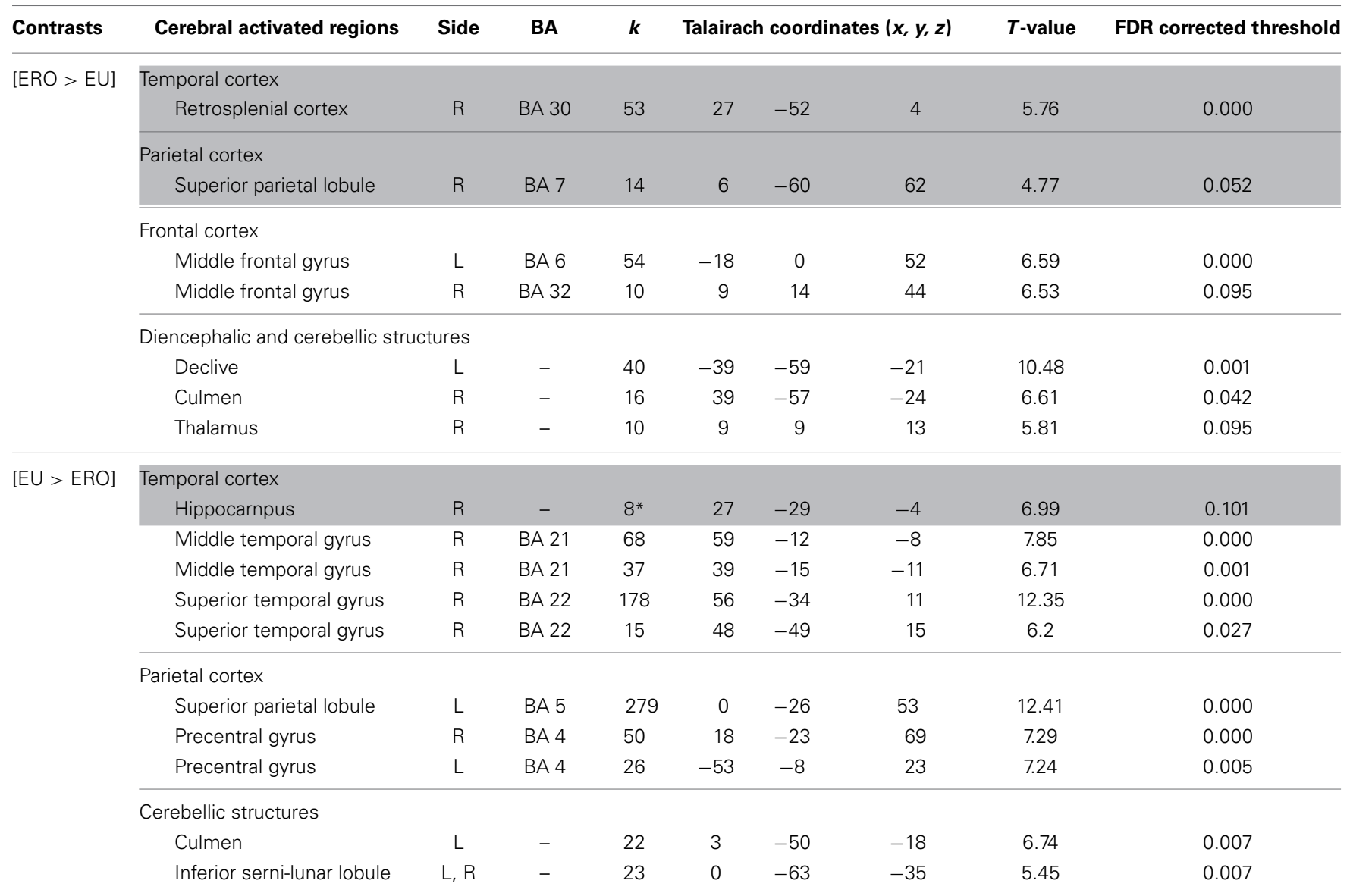

They were provided by the contrast: (1) ERO vs. EU for the upper part of the table. (2) EU vs. ERO for the lower part of the table (random-effect analysis, uncorrected $p<0.001, k \geq 10$ voxels, ${ }^{*}$ for the hippocampus $\left.k=8\right)$. The Talairach coordinates $(x, y, z)$ are indicated for each voxel. The side, Right (R) and Left (L), gyri and Brodmann areas (BA) are mentioned. FDR corrected threshold provides corrected values detailing the prevalence of false positives in the analysis.

This result therefore provides evidence that the retrosplenial cortex in humans specifically processes changes in head direction. Moreover, although the performances for A and EU conditions were correlated, the ERO performance was not correlated with the allocentric or the egocentric-updated performance. Therefore, participants that were good at processing head direction changes were not necessarily good at processing location changes or allocentric landmark-based information. This supports the idea that relatively independent cognitive mechanisms are at play in those tasks.

Although the retrosplenial cortex (BA 30) is known to contain head-direction cells in rodents (Cho and Sharp, 2001; Sharp et al., 2001; Wiener and Taube, 2005), only limited experimental evidence was reported in humans. Concerning clinical evidence, it has been suggested that right retrosplenial lesions lead to pure topographical disorientations (Valenstein et al., 1987; Yasuda et al., 1997; Aguirre and D’Esposito, 1999; Maguire, 2001; Vann and Aggleton, 2004; Vann et al., 2009), reflecting a type of heading disorientation (i.e., an inability to represent the orientation direction with respect to the external environment).
Functional MRI studies have provided evidence suggesting that the retrosplenial cortex was strongly activated during scene viewing, scene imagery, and scene memory (Epstein et al., 2007). Moreover, a meta-analysis of navigation fMRI studies reported bilateral involvement of BA 30 in humans (Maguire, 2001; for an extensive review see Vann et al., 2009). Congruently, we report a common spatial encoding network encompassing the bilateral retrosplenial cortex (when compared to control condition). Overall, these results make it possible to pinpoint the implication of the retrosplenial region in spatial processing.

However, with regards to head-direction, only one fMRI study aimed at identifying neural correlates of orientation This study reported a region situated close to the anatomically defined retrosplenial cortex (BA 30, see Maguire, 2001, for a review), in BA 31 (Baumann and Mattingley, 2010). In our current study we report for the first time the activation of the right retrosplenial cortex (BA 30) for the rotation. This result is in agreement with animal studies of spatial encoding. Additionally, we observed the BA 30 activation during ERO vs. A contrast, next to the bilateral posterior cingulate (BA 31), in line with Baumann and Mattingley (2010)'s findings. 


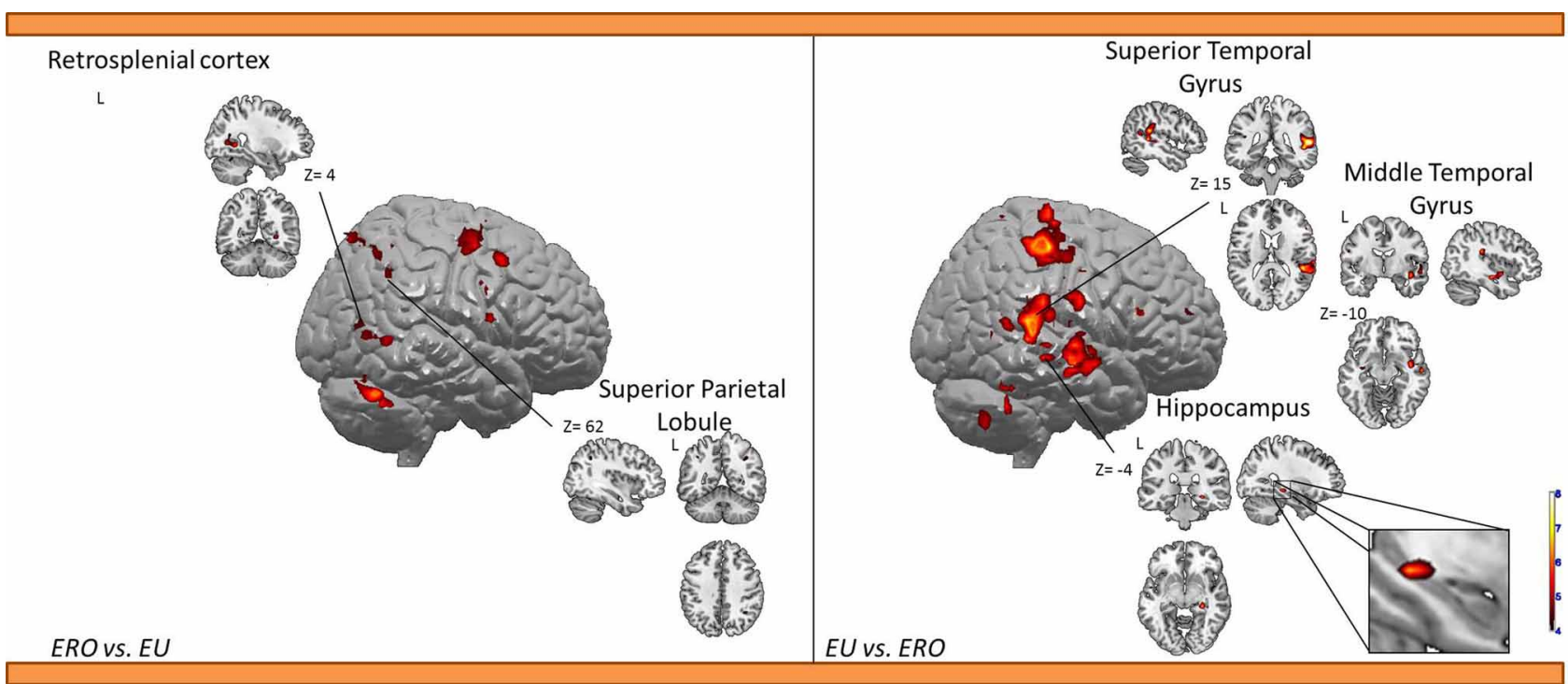

FIGURE 3 | Shows regions reported in Table 4 projected onto a 3D anatomical template (random-effect analysis, statistical threshold: uncorrected $\boldsymbol{p}<\mathbf{0 . 0 0 1}$, cluster extent: $\boldsymbol{k} \geq \mathbf{1 0}$ voxels). On the left, ERO is contrasted to EU; on the right, EU is contrasted to ERO. The retrosplenial activity, along with the superior parietal gyrus activations are highlighted for the right contrast. The right hippocampal activity (cluster extent $k=8$ voxels) along with the superior and inferior temporal gyrus activations are highlighted on the right contrast. These clusters are projected onto 2D anatomical slices (T1-template image) in neurological convention. $L$ is left hemisphere. The color scale represents the $t$-value of activation.

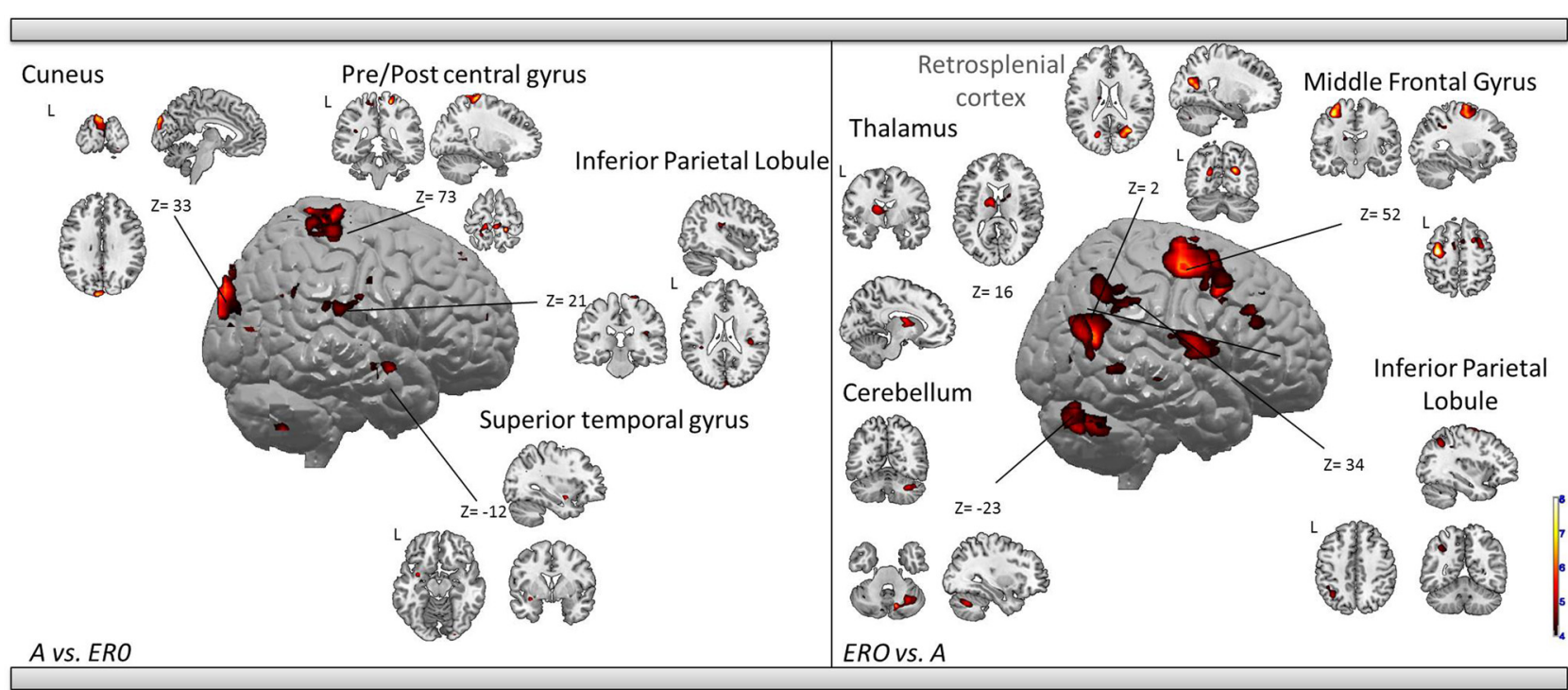

FIGURE 4 | Shows regions reported in Table 3 projected onto a 3D anatomical template (random-effect analysis, statistical threshold: uncorrected $p<\mathbf{0 . 0 0 1}$, cluster extent: $\boldsymbol{k} \geq \mathbf{1 0}$ voxels). Activations are provided by a random effect analysis, for the A spatial encoding when contrasted (1) with the EU condition (on the left), and (2) with the
ERO condition (on the right). Occipital activity in the cuneus which is observed in both contrasts is highlighted. These clusters are projected onto 2D anatomical slices (T1-template image) in neurological convention. $\mathrm{L}$ is left hemisphere. The color scale represents the $t$-value of activation.
An influential model of spatial memory proposes that the retrosplenial cortex supports, in humans, stimulus conversion from an egocentric reference frame (in the parietal cortex) to an allocentric reference frame (in the medial temporal regions) and vice versa (Burgess et al., 2001a,b; Burgess, 2008; Vann et al.,
2009). However, with regards to retrosplenial function, this model was mainly based on animal studies evidence. Few fMRI studies have reported retrosplenial activity congruent with this model (Lambrey et al., 2012; Zhang et al., 2012). Our result supports the hypothesis of retrosplenial cortex encompassing cells activated 
Table 5 | Activated regions provided by the contrast: (1) A vs. ERO for the upper part of the table; (2) ERO vs. A for the lower part of the table (random-effect analysis, uncorrected $p<0.001, k \geq 10$ voxels).

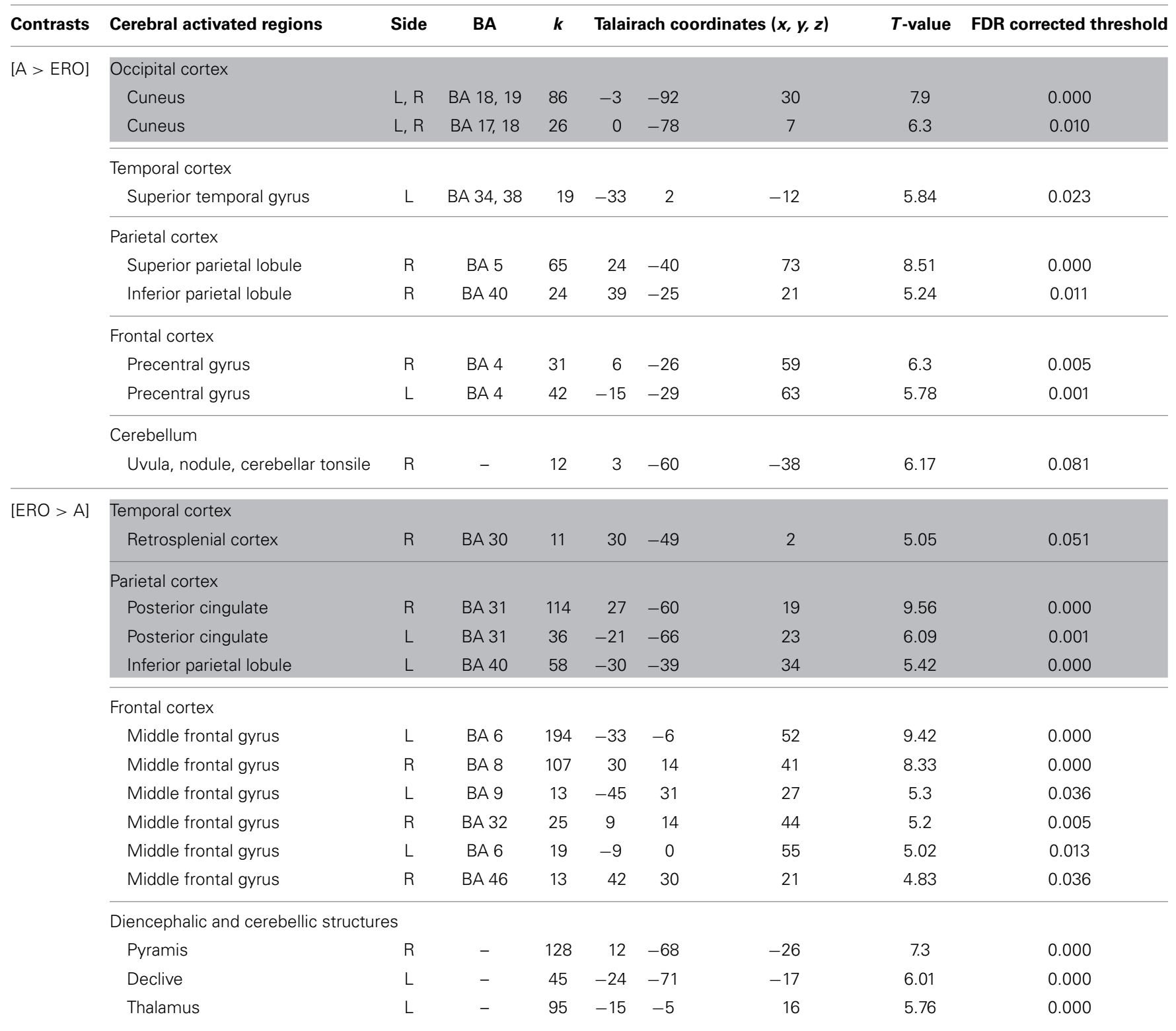

The Talairach coordinates $(x, y, z)$ are indicated for each voxel. The Hemisphere, Right (R) and Left (L), gyri and Brodmann areas (BA) are mentioned. Regions reported in both contrasts are highlighted. FDR corrected threshold provides corrected values detailing the prevalence of false positives in the analysis.

Table 6 | Hippocampal and retrosplenial activation in the FDR small-volume corrected analysis $(p<0.05)$ of spatial encoding conditions.

\begin{tabular}{llccrrr}
\hline Contrasts & Cerebral regions & Side & BA & & Talairach coordinates $(\boldsymbol{x}, \boldsymbol{y}, \boldsymbol{z})$ & $\boldsymbol{T}$-value \\
\hline$[E U>A]$ & Hippocampus & $R$ & - & 30 & -23 & -5 \\
{$[E R O>E U]$} & Retrosplenial cortex & $R$ & BA 30 & 24 & -52 & 0 \\
{$[E U>E R O]$} & Hippocampus & $R$ & - & 24 & -29 & -4 \\
{$[E R O>A]$} & Retrosplenial cortex & R & BA 30 & 15 & -59 & 9 \\
& Retrosplenial cortex & R & BA 31 & 12 & -68 & 21 \\
\hline
\end{tabular}

The Talairach coordinates $(x, y, z)$ are indicated for each voxel. The Hemisphere, Right $(R)$ and Left $(L)$, gyri and Brodmann areas (BA) are mentioned. 
for head-direction in humans. Neuronal electrical recordings in humans are necessary to provide further direct support of the retrosplenial involvement.

The second crucial activated region reported in this study is the right hippocampal area. It was preferentially involved in spatial encoding, which required an online integration of self-location transformation in the spatial representation, rather than encoding the schematic spatial relation amongst objects (O'Keefe and Nadel, 1978). In fact, the contrast of the EU condition to the A condition significantly enhanced the right hippocampal activity. This result is in line with previous observations of right hippocampal activation during path integration encoding in humans (Wolbers et al., 2007). Nonetheless, during retrieval, the left hippocampal structure might be further involved, as suggested by Iglói et al. (2010). Moreover, this hippocampal activation is congruent with Shelton and Gabrieli (2002)'s results which compared cerebral activities during encoding from a route perspective and a survey perspective. They observed bilateral hippocampal activation specific to the route condition. Differences in the type of subsequent task-demand (recognition task vs. spatial self-toobject pointing in this study) and the environment complexity (large with multiple boundaries and 17 landmarks vs. medium with an average of 5 landmarks in this study) can account for the right lateralization of the hippocampal activation in the present study.

Moreover, the modulation by between-subject retrieval performance of a hippocampal-caudate cluster observed in the correlation analysis indicates a critical role for these structures in the subsequent task-resolution. During encoding, good egocentric-updating pointers showed stronger hippocampal activation (extending to caudate). The hippocampal activity has previously been reported to correlate to accuracy of self-location or self-orientation, in a path integration task (Wolbers et al., 2007, using an egocentric pointing task) or during active navigation, (Maguire et al., 1998; Hartley et al., 2003). We therefore interpret this hippocampal activity in good memory performers as highlighting the importance of the right hippocampus for processing self-location changes.

Because the EU vs. A contrast provides the neural substrate engaged by the egocentric-updating and due to self-motion information (i.e., idiothetic) rather than landmark-based information, we suggest that the right hippocampal region may be more specifically devoted to location related self-related changes. Additionally, because allocentric processing such as landmarks and room geometry processing were likely to occur in the EU condition as well, it is possible that the hippocampal involvement could also arise from the relative flexibility of the representation constituted during the EU encoding compared to the more schematic representation during the A encoding. In fact, Zhang and Ekstrom (2013) have shown that such differences in the use of an allocentric representation could be responsible for a differential hippocampal involvement in favor of a flexible representation. As this right hippocampal activity is also observed when directly contrasting EU vs. ERO within egocentric referencing, it is likely that self-motion location change in the EU condition plays a crucial role in this hippocampal activation. This additional evidence supports the hypothesis that, in humans, location self-related changes involve the right hippocampal region.

This result is new and central, as a wide body of evidence from animal literature, and in particular the discovery of place cells, have led to the proposal that the hippocampal structure underlies cognitive mapping in an allocentric reference frame (O'Keefe and Dostrovsky, 1971; O'Keefe and Nadel, 1978; O'Keefe et al., 1998). In humans, single cells neuron recordings have shown that place cells exist in the human hippocampus (Ekstrom et al., 2003).

In the present experiment, we report substantial hippocampal activation when participants must encode allocentric landmarkbased spatial information (there is a significant activation of the hippocampus in the A encoding condition compared to the $\mathrm{C}$ encoding condition). However, given previous neuropsychological results, we expected that the hippocampal structure could be enhanced when idiothetic self-location changes are involved (Worsley et al., 2001; Philbeck et al., 2004; Gomez et al., 2012). From a behavioral perspective, participants who performed well in the EU condition also performed well in the A condition. Together with the conjunction results, this observation supports the hypothesis that a unique structure, the hippocampus, may underlie different spatial processing, in the present case, egocentric-updating and allocentric processing, which could explain this correlational effect.

Overall, these findings support models emphasizing the need to combine allocentric landmark sensory aspects to egocentricupdating to form the place code of Place cells (e.g., Redish and Touretzky, 1997). Several proposals have suggested that the hippocampus might be involved in complementary types of spatial processing such as path integration processing (Redish and Touretzky, 1997; Whishaw et al., 1997, 2001; Worsley et al., 2001; Wolbers et al., 2007). These models have underlined that the hippocampal structure is well-suited to underlie such spatial processing as it is interconnected to various structures making it possible to represent, together, the body's position: it receives input from systems assumed to represent head-direction (such as the retrosplenial cortex, and thalamus), and from systems processing self-motion, such as the human motion complex (Redish and Touretzky, 1997; Wiener and Taube, 2005).

In closing, our results refine previous findings on navigation in large-scale environments (Maguire et al., 1998; Ekstrom et al., 2003; Hartley et al., 2003) by suggesting that hippocampal activity should be extended to represent self-related location transformations. In keeping with existing models (Burgess et al., 2001a; Buzsaki, 2005), we suggest that, in humans, during spatial encoding: (1) the retrosplenial cortex processes headingdirection; (2) the hippocampus processes self-related location transformations and combines it with landmark information to allow place computations. Such a combination would allow for correcting accumulated errors during egocentric-updating.

To conclude, this experiment highlighted that, in some circumstances determined by the experimental conditions, hippocampal and retrosplenial structures known to be involved in allocentric environmental coding (Galati et al., 2010) could demonstrate a preferential involvement in an egocentric coding of space. Consequently the differentiation between allocentric vs. egocentric representation no longer seems to be sufficient in 
understanding the complexity of the mechanisms at play during spatial encoding.

\section{SUPPLEMENTARY MATERIAL}

The Supplementary Material for this article can be found online at: http://www.frontiersin.org/journal/10.3389/fnhum. 2014.00150/abstract

\section{REFERENCES}

Aguirre, G. K., and D'Esposito, M. (1999). Topographical disorientation: a synthesis and taxonomy. Brain 122(Pt 9), 1613-1628.

Aguirre, G. K., Detre, J. A., Alsop, D. C., and D'Esposito, M. (1996). The parahippocampus subserves topographical learning in man. Cereb. Cortex 6, 823-829.

Allen, G. L. (1999). "Spatial abilities, cognitive maps, and wayfinding: bases for individual differences in spatial cognition and behavior," in Wayfinding Behavior: Cognitive Mapping and Other Spatial Processes, ed R. G. Golledge (Baltimore, MD: Johns Hopkins Press), 46-80.

Andersen, R. A., Essick, G. K., and Siegel, R. M. (1985). Encoding of spatial location by posterior parietal neurons. Science 230, 456-458. doi: 10.1126/science. 4048942

Bastin, J., Committeri, G., Kahane, P., Galati, G., Minotti, L., Lachaux, J.-P., et al. (2013). Timing of posterior parahippocampal gyrus activity reveals multiple scene processing stages. Hum. Brain Mapp. 34, 1357-1370. doi: 10.1002/hbm. 21515

Baumann, O., Chan, E., and Mattingley, J. B. (2010). Dissociable neural circuits for encoding and retrieval of object locations during active navigation in humans. Neuroimage 49, 2816-2825. doi: 10.1016/j.neuroimage.2009.10.021

Baumann, O., and Mattingley, J. B. (2010). Medial parietal cortex encodes perceived heading direction in humans. J. Neurosci. 30, 12897. doi: 10.1523/JNEUROSCI.3077-10.2010

Bennett, C. M., Wolford, G. L., and Miller, M. B. (2009). The principled control of false positives in neuroimaging. Soc. Cogn. Affect. Neurosci. 4, 417-422. doi: 10.1093/scan/nsp053

Bisiach, E., and Luzzatti, C. (1978). Unilateral neglect of representational space. Cortex 14, 129-133. doi: 10.1016/S0010-9452(78)80016-1

Bohbot, V. D., Kalina, M., Stepankova, K., Spackova, N., Petrides, M., and Nadel, L. Y. N. N. (1998). Spatial memory deficits in patients with lesions to the right hippocampus and to the right parahippocampal cortex. Neuropsychologia 36, 1217-1238. doi: 10.1016/S0028-3932(97)00161-9

Burgess, N. (2006). Spatial memory: how egocentric and allocentric combine. Trends Cogn. Sci. (Regul. Ed). 10, 551-557. doi: 10.1016/j.tics.2006.10.005

Burgess, N. (2008). Spatial cognition and the brain. Ann. N.Y. Acad. Sci. 1124, 77-97. doi: 10.1196/annals.1440.002

Burgess, N., Becker, S., King, J. A., and O'Keefe, J. (2001a). Memory for events and their spatial context: models and experiments. Philosophical Transcript of the Royal Society 356, 1493-1503. doi: 10.1098/rstb.2001.0948

Burgess, N., Maguire, E. A., Spiers, H. J., and O'Keefe, J. (2001b). A temporoparietal and prefrontal network for retrieving the spatial context of lifelike events. Neuroimage 14, 439-453. doi: 10.1006/nimg.2001.0806

Burgess, N., Trinkler, I., King, J., Kennedy, A., and Cipolotti, L. (2006). Impaired allocentric spatial memory underlying topographical disorientation. Rev. Neurosci. 17, 239-251. doi: 10.1515/REVNEURO.2006.17.1-2.239

Buzsaki, G. (2005). Theta rhythm of navigation: link between path integration and landmark navigation, episodic and semantic memory. Hippocampus 15, 827-840. doi: 10.1002/hipo.20113

Cho, J., and Sharp, P. E. (2001). Head direction, place, and movement correlates for cells in the rat retrosplenial cortex. Behav. Neurosci. 115:3. doi: 10.1037/07357044.115.1.3

Chokron, S. (2003). Right parietal lesions, unilateral spatial neglect, and the egocentric frame of reference. Neuroimage 20, 75-81. doi: 10.1016/j.neuroimage.2003.09.002

Committeri, G., Galati, G., Paradis, A. L., Pizzamiglio, L., Berthoz, A., and LeBihan, D. (2004). Reference frames for spatial cognition: different brain areas are involved in viewer-, object-, and landmark-centered judgments about object location. J. Cogn. Neurosci. 16, 1517-1535. doi: 10.1162/08989290425 68550
Ekstrom, A. D., Kahana, M. J., Caplan, J. B., Fields, T. A., Isham, E. A., Newman, E. L., et al. (2003). Cellular networks underlying human spatial navigation. Nature 425, 184-188. doi: 10.1038/nature01964

Epstein, R. A., Parker, W. E., and Feiler, A. M. (2007). Where am I now? Distinct roles for parahippocampal and retrosplenial cortices in place recognition. $J$. Neurosci. 27, 6141-6149. doi: 10.1523/JNEUROSCI.0799-07.2007

Farrell, M. J., and Robertson, I. H. (1998). Mental rotation and automatic updating of body-centered spatial relationships. J. Exp. Psychol. Learn. Mem. Cogn. 24:227. doi: 10.1037/0278-7393.24.1.227

Fields, A. W., and Shelton, A. L. (2006). Individual skill differences and large-scale environmental learning. J. Exp. Psychol. Learn. Mem. Cogn. 32, 506-515. doi: 10.1037/0278-7393.32.3.506

Finke, C., Ostendorf, F., Braun, M., and Ploner, C. J. (2011). Impaired representation of geometric relationships in humans with damage to the hippocampal formation. PLoS ONE 6:e19507. doi: 10.1371/journal.pone.0019507

Friston, K. J., Fletcher, P., Josephs, O., Holmes, A., Rugg, M. D., and Turner, R. (1998). Event-related fMRI: characterizing differential responses. Neuroimage 7, 30-40. doi: 10.1006/nimg.1997.0306

Friston, K. J., Penny, W. D., and Glaser, D. E. (2005). Conjunction revisited. Neuroimage 25, 661-667. doi: 10.1016/j.neuroimage.2005.01.013

Galati, G., Lobel, E., Vallar, G., Berthoz, A., Pizzamiglio, L., and Le Bihan, D. (2000). The neural basis of egocentric and allocentric coding of space in humans: a functional magnetic resonance study. Exp. Brain Res. 133, 156-164. doi: $10.1007 / \mathrm{s} 002210000375$

Galati, G., Pelle, G., Berthoz, A., and Committeri, G. (2010). Multiple reference frames used by the human brain for spatial perception and memory. Exp. Brain Res. 206, 109-120. doi: 10.1007/s00221-010-2168-8

Ghaem, O., Mellet, E., Crivello, F., Tzourio, N., Mazoyer, B., Berthoz, A., et al. (1997). Mental navigation along memorized routes activates the hippocampus, precuneus, and insula. Neuroreport 8, 739-744. doi: 10.1097/00001756199702100-00032

Gomez, A. (2014a). Allocentric video example. doi: 10.6084/m9.figshare.902846

Gomez, A. (2014b). Egocentric-updating video example. doi: $10.6084 / \mathrm{m} 9$. figshare. 902847

Gomez, A. (2014c). Egocentric with Head-rotation video example. doi: $10.6084 / \mathrm{m} 9$. figshare. 902848

Gomez, A., Rousset, S., Bonniot, C., Charnallet, A., and Moreaud, O. (2014). Deficits in egocentric-updating and spatial context memory in a case of developmental amnesia. Neurocase doi: 10.1080/13554794.2014.890730. [Epub ahead of print].

Gomez, A., Rousset, S., and Charnallet, A. (2012). Spatial deficits in an amnesic patient with hippocampal damage: questioning the multiple trace theory. Hippocampus 22, 1313-1324. doi: 10.1002/hipo.20968

Hartley, T., Maguire, E. A., Spiers, H. J., and Burgess, N. (2003). The well-worn route and the path less traveled: distinct neural bases of route following and wayfinding in humans. Neuron 37, 877-888. doi: 10.1016/S08966273(03)00095-3

Holdstock, J. S., Mayes, A. R., Cezayirli, E., Isaac, C. L., Aggleton, J. P., and Roberts, N. (2000). A comparison of egocentric and allocentric spatial memory in a patient with selective hippocampal damage. Neuropsychologia 38, 410-425. doi: 10.1016/S0028-3932(99)00099-8

Iglói, K., Doeller, C. F., Berthoz, A., Rondi-Reig, L., and Burgess, N. (2010). Lateralized human hippocampal activity predicts navigation based on sequence or place memory. Proc. Natl. Acad. Sci. U.S.A. 107, 14466-14471. doi: 10.1073/pnas. 1004243107

Jacobs, J., Kahana, M. J., Ekstrom, A. D., Mollison, M. V., and Fried, I. (2010). A sense of direction in human entorhinal cortex. Proc. Natl. Acad. Sci. U.S.A. 107, 6487-6492. doi: 10.1073/pnas.0911213107

Janzen, G., and Van Turennout, M. (2004). Selective neural representation of objects relevant for navigation. Nat. Neurosci. 7, 673-677. doi: 10.1038/nn1257

Janzen, G., Wagensveld, B., and Van Turennout, M. (2007). Neural representation of navigational relevance is rapidly induced and long lasting. Cereb. Cortex 17, 975. doi: $10.1093 /$ cercor/bhl008

Janzen, G., and Weststeijn, C. G. (2007). Neural representation of object location and route direction: an event-related fMRI study. Brain Res. 1165, 116-125. doi: 10.1016/j.brainres.2007.05.074

Lambrey, S., Doeller, C., Berthoz, A., and Burgess, N. (2012). Imagining being somewhere else: neural basis of changing perspective in space. Cereb. Cortex 22, 166-174. doi: 10.1093/cercor/bhr101 
Lancaster, J. L., Woldorff, M. G., Parsons, L. M., Liotti, M., Freitas, C. S., Rainey, L., et al. (2000). Automated Talairach atlas labels for functional brain mapping. Hum. Brain Mapp. 10, 120-131. doi: 10.1002/10970193(200007)10:3\%3C120::AID-HBM30\%3E3.0.CO;2-8

Leutgeb, S., Ragozzino, K. E., and Mizumori, S. J. (2000). Convergence of head direction and place information in the CA1 region of hippocampus. Neuroscience 100, 11-20. doi: 10.1016/S0306-4522(00)00258-X

Maguire, E. A. (2001). The retrosplenial contribution to human navigation: a review of lesion and neuroimaging findings. Scand. J. Psychol. 42, 225-238. doi: 10.1111/1467-9450.00233

Maguire, E. A., Burgess, N., Donnett, J. G., Frackowiak, R. S., Frith, C. D., and O'Keefe, J. (1998). Knowing where and getting there: a human navigation network. Science 280, 921-924.

Maguire, E. A., Nannery, R., and Spiers, H. J. (2006). Navigation around London by a taxi driver with bilateral hippocampal lesions. Brain 129(Pt 11), 2894-2907. doi: 10.1093/brain/awl286

McNaughton, B. L., Battaglia, F. P., Jensen, O., Moser, E. I., and Moser, M. B. (2006). Path integration and the neural basis of the'cognitive map'. Nat. Rev. Neurosci. 7, 663-678. doi: 10.1038/nrn1932

Mellet, E., Briscogne, S., Tzourio-Mazoyer, N., Ghaem, O., Petit, L., Zago, L., et al. (2000). Neural correlates of topographic mental exploration: the impact of route versus survey perspective learning. Neuroimage 12, 588-600. doi: 10.1006/nimg.2000.0648

Milner, A. D., and Goodale, M. A. (2008). Two visual systems re-viewed. Neuropsychologia 46, 774-785. doi: 10.1016/j.neuropsychologia.2007.10.005

Mittelstaedt, H., and Mittelstaedt, M. (1982). Homing by Path Integration. Heidelberg: Springer-Verlag.

O'Keefe, J., and Burgess, N. (1996). Geometric determinants of the place fields of hippocampal neurons. Nature 381, 425-428.

O'Keefe, J., Burgess, N., Donnett, J. G., Jeffery, K. J., and Maguire, E. A. (1998). Place cells, navigational accuracy, and the human hippocampus. Philos. Trans. R. Soc. Lond. B Biolol. Sci. 353, 1333-1340.

O'Keefe, J., and Dostrovsky, J. (1971). The hippocampus as a spatial map. Preliminary evidence from unit activity in the freely-moving rat. Brain Res. 34 171-175.

O'Keefe, J., and Nadel, L. (1978). The Hippocampus as a Cognitive Map. London: Oxford University Press.

Oldfield, R. C. (1971). The assessment and analysis of handedness: the Edinburgh inventory. Neuropsychologia 9, 97-113. doi: 10.1016/0028-3932(71)90067-4

Philbeck, J. W., Behrmann, M., Levy, L., Potolicchio, S. J., and Caputy, A. J. (2004) Path integration deficits during linear locomotion after human medial temporal lobectomy. J. Cogn. Neurosci. 16, 510-520. doi: 10.1162/089892904323057254

Ranck, J. B. Jr. (1984). Head direction cells in the deep cell layer of dorsal presubiculum in freely moving rats. Soc. Neurosci. Abstr. 10:176.12.

Redish, A. D., and Touretzky, D. S. (1997). Cognitive maps beyond the hippocampus. Hippocampus 7, 15-35.

Robertson, R. G., Rolls, E. T., Georges-François, P., and Panzeri, S. (1999). Head direction cells in the primate pre-subiculum. Hippocampus 9, 206-219.

Sharp, P. E., Blair, H. T., and Cho, J. (2001). The anatomical and computational basis of the rat head-direction cell signal. Trends Neurosci. 24, 289-294. doi: 10.1016/S0166-2236(00)01797-5

Shelton, A. L., and Gabrieli, J. D. (2002). Neural correlates of encoding space from route and survey perspectives. J. Neurosci. 22, 2711-2717.

Shelton, A. L., and Gabrieli, J. D. (2004). Neural correlates of individual differences in spatial learning strategies. Neuropsychology 18, 442-449. doi: 10.1037/08944105.18.3.442

Shelton, A. L., and Pippitt, H. A. (2007). Fixed versus dynamic orientations in environmental learning from ground-level and aerial perspectives. Psychol. Res. 71, 333-346. doi: 10.1007/s00426-006-0088-9

Shrager, Y., Kirwan, C. B., and Squire, L. R. (2008). Neural basis of the cognitive map: path integration does not require hippocampus or entorhinal cortex. Proc. Natl. Acad. Sci. U.S.A. 105, 12034-12038. doi: 10.1073/pnas.0805414105

Spiers, H. J., Burgess, N., Maguire, E. A., Baxendale, S. A., Hartley, T., Thompson, P. J., et al. (2001). Unilateral temporal lobectomy patients show lateralized topographical and episodic memory deficits in a virtual town. Brain 124(Pt 12), 2476-2489. doi: 10.1093/brain/124.12.2476

Talairach, J., and Tournoux, P. (1988). Co-planar Stereotaxic Atlas of the Human Brain. 3-Dimensional Proportional System: an Approach to Cerebral Imaging. New York, NY: Thieme Medical Publisher.
Taube, J. S. (1998). Head direction cells and the neurophysiological basis for a sense of direction. Prog. Neurobiol. 55, 225-256. doi: 10.1016/S0301-0082(98)00004-5

Thorndyke, P. W., and Hayes-Roth, B. (1982). Differences in spatial knowledge acquired from maps and navigation. Cogn. Psychol. 14, 560-589. doi: 10.1016/0010-0285(82)90019-6

Valenstein, E., Bowers, D., Verfaellie, M., Heilman, K. M., Day, A., and Watson, R. T. (1987). Retrosplenial amnesia. Brain 110:1631. doi: 10.1093/brain/110.6.1631

Vallar, G., Lobel, E., Galati, G., Berthoz, A., Pizzamiglio, L., and Le Bihan, D. (1999). A fronto-parietal system for computing the egocentric spatial frame of reference in humans. Exp. Brain Res. 124, 281-286. doi: 10.1007/s002210050624

Vann, S. D., and Aggleton, J. P. (2004). Testing the importance of the retrosplenia guidance system: effects of different sized retrosplenial cortex lesions on heading direction and spatial working memory. Behav. Brain Res. 155, 97-108. doi: 10.1016/j.bbr.2004.04.005

Vann, S. D., Aggleton, J. P., and Maguire, E. A. (2009). What does the retrosplenial cortex do? Nat. Rev. Neurosci. 10, 792-802. doi: 10.1038/nrn2733

Whishaw, I. Q., Hines, D. J., and Wallace, D. G. (2001). Dead reckoning (path integration) requires the hippocampal formation: evidence from spontaneous exploration and spatial learning tasks in light (allothetic) and dark (idiothetic) tests. Behav. Brain Res. 127, 49-69. doi: 10.1016/S0166-4328(01)00359-X

Whishaw, I. Q., McKenna, J. E., and Maaswinkel, H. (1997). Hippocampal lesions and path integration. Curr. Opin. Neurobiol. 7, 228-234. doi: 10.1016/S09594388(97)80011-6

Wiener, S. I., and Taube, J. S. (2005). Head Direction Cells and the Neural Mechanisms of Spatial Orientation (Bradford Books). Cambridge, MA: The MIT press.

Wolbers, T., and Buchel, C. (2005). Dissociable retrosplenial and hippocampal contributions to successful formation of survey representations. J. Neurosci. 25, 3333. doi: 10.1523/JNEUROSCI.4705-04.2005

Wolbers, T., and Hegarty, M. (2010). What determines our navigational abilities? Trends Cogn. Sci. 14, 138-146. doi: 10.1016/j.tics.2010.01.001

Wolbers, T., Hegarty, M., Büchel, C., and Loomis, J. M. (2008). Spatial updating: how the brain keeps track of changing object locations during observer motion. Nat. Neurosci. 11, 1223-1230. doi: 10.1038/nn.2189

Wolbers, T., Wiener, J. M., Mallot, H. A., and Buchel, C. (2007). Differential recruitment of the hippocampus, medial prefrontal cortex, and the human motion complex during path integration in humans. J. Neurosci. 27, 9408-9416. doi: 10.1523/JNEUROSCI.2146-07.2007

Worsley, C. L., Recce, M., Spiers, H. J., Marley, J., Polkey, C. E., and Morris, R. G. (2001). Path integration following temporal lobectomy in humans. Neuropsychologia 39, 452-464. doi: 10.1016/S0028-3932(00)00140-8

Yasuda, Y., Watanabe, T., Tanaka, H., Tadashi, I., and Akiguchi, I. (1997). Amnesia following infarction in the right retrosplenial region. Clin. Neurol. Neurosurg 99, 102-105. doi: 10.1016/S0303-8467(97)80005-4

Zhang, H., Copara, M., and Ekstrom, A. D. (2012). Differential recruitment of brain networks following route and cartographic map learning of spatial environments. PLoS ONE 7:e44886. doi: 10.1371/journal.pone.00 44886

Zhang, H., and Ekstrom, A. (2013). Human neural systems underlying rigid and flexible forms of allocentric spatial representation. Hum. Brain Mapp. 34, 1070-1087. doi: 10.1002/hbm.21494

Conflict of Interest Statement: The authors declare that the research was conducted in the absence of any commercial or financial relationships that could be construed as a potential conflict of interest.

Received: 22 November 2013; accepted: 27 February 2014; published online: 20 March 2014.

Citation: Gomez A, Cerles M, Rousset S, Rémy C and Baciu M (2014) Differential hippocampal and retrosplenial involvement in egocentric-updating, rotation, and allocentric processing during online spatial encoding: an fMRI study. Front. Hum. Neurosci. 8:150. doi: 10.3389/fnhum.2014.00150

This article was submitted to the journal Frontiers in Human Neuroscience.

Copyright (C) 2014 Gomez, Cerles, Rousset, Rémy and Baciu. This is an open-access article distributed under the terms of the Creative Commons Attribution License (CC BY). The use, distribution or reproduction in other forums is permitted, provided the original author(s) or licensor are credited and that the original publication in this journal is cited, in accordance with accepted academic practice. No use, distribution or reproduction is permitted which does not comply with these terms. 